\title{
Space-Time Interpolation and Automatic Mapping of TEC Using TNPGN-Active
}

\author{
Feza Arikan ${ }^{1}$, Orhan Arikan $^{2}$, Umut Sezen $^{1}$, Cenk Toker ${ }^{1}$, Bahadir Aktug $^{3}$, Onur Lenk $^{3}$, Mustafa Kurt ${ }^{3}$ and Erdem \\ Parmaksiz ${ }^{3}$
}

${ }^{1}$ Hacettepe University, Department of Electrical and Electronics Engineering, Beytepe, Ankara 06800, Turkey

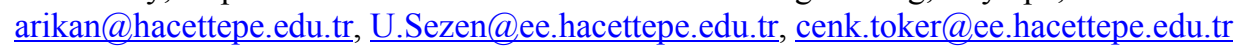

${ }^{2}$ Bilkent University, Department of Electrical and Electronics Engineering, Bilkent, Ankara 06800 Turkey oarikan@ee.bilkent.edu.tr

${ }^{3}$ General Command of Mapping, Defense Ministry, Cebeci, Ankara Turkey bahadir.aktug@hgk.msb.gov.tr, onur.lenk@hgk.msb.gov.tr, mustafa.kurt@hgk.msb.gov.tr, erdem.parmaksiz@hgk.msb.gov.tr

\begin{abstract}
Turkish National Permanent GPS Network (TNPGN) is the Reference Station Network of 146 continuouslyoperating GNSS stations o which are distributed uniformly across Turkey and North Cyprus Turkish Republic since May 2009. IONOLAB group, formed by researchers and students in Hacettepe University, Bilkent University and General Command of Mapping is currently investigating new techniques for space-time interpolation, and automatic mapping of TEC through a TUBITAK research grant. This study presents the developments in monitoring of space weather, and correction of geodetic positioning errors due to ionosphere using TNPGN.
\end{abstract}

\section{Introduction}

Ionosphere is the main source of positioning error for GNSS receivers due to its high spatial and temporal variability. It is of utmost importance to estimate and predict the variability in space weather and compute the signal delays due to the ionospheric and plasmaspheric frequency dependent refractivity. TNPGN consisting of 146 GNSS stations which are homogenously distributed over Turkey and North Cyprus Turkish Republic, has been recently established. TNPGN has been operational since May 2009 and it provides a wide variety of possibilities for active research not only in geodesy but also in the fields ranging from geology to the tropospheric and ionospheric characterization. IONOLAB is a group of researchers of various disciplines, getting together to handle challenges of the Earth's ionosphere. The team has researchers from Hacettepe University and Bilkent University, Departments of Electrical and Electronics Engineering, and General Command of Mapping. IONOLAB group has been active in the estimation of Total Electron Content (TEC) by using single station GPS recordings in the IGS network [1-4], regional spatial interpolation (mapping) of TEC [5-6], global Computerized Ionospheric Tomography (CIT) [7-9], statistical analysis of TEC [10-11], and the investigation of lithosphere-ionosphere coupling through seismic activity [12]. The techniques that have been developed for various purposes can be applied to any GNSS station in IGS or EUREF network. With the new TNPGN, it is observed that there are various prospective areas in the data processing GNSS measurements for higher accuracy and reliability in the characterization of the regional ionosphere. The first one of these areas is an efficient and effective signal processing algorithm for computation of TEC using 146 GNSS stations continuously operating in a network. A new technique is developed to recover TEC data gaps utilizing a weighted time and space interpolation. The second task is the monitoring of the TEC using optimized and automatic spatial interpolation, i.e. mapping. The east-west extend of Turkey indicates a major anisotropy in TEC distribution especially during sunrise and sunset hours. A north-south anisotropy is also observed for hours with full sun exposure and during night hours. IONOLAB group is currently investigating new techniques for incorporating anisotropicity into Ordinary Kriging for TEC mapping and generation of an accurate temporal model for the semivariogram function for TNPGN. In this study, these goals and the developments will be discussed in the following sections.

\section{Space-Time Interpolation of TEC}

GPS signals are affected by multipath, environmental or physical conditions and various disruptions of data or problems like cycle slips may occur in the estimation of TEC. This situation causes TEC to be computed in discrete epochs. Since most of these shortage periods are due to the factors outside ionosphere as listed above, the gaps in estimated TEC should be recovered. A novel algorithm is developed by IONOLAB group that combines temporal 
interpolation with spatial interpolation employing pre-determined weight coefficients. The Cubic Spline (C-Spline) is chosen as the temporal interpolation method. For spatial interpolation, GNSS reference stations within $200 \mathrm{~km}$ neighborhood are chosen and a linear regression algorithm similar to Ordinary Kriging is employed. The weights are chosen according to the level of disturbance in the ionosphere and the length of data loss. If the data loss is less than one hour, the temporal interpolation has higher weight and for durations longer than one hour, spatial interpolation dominates. Figure 1a denotes a histogram of number of stations that has discontinuities in IONOLAB-TEC. A further analysis indicates that most of data loss occurs for durations shorter than two hours. The technique is demonstrated for the IONOLAB-TEC values of Adana station $\left(37^{\circ} \mathrm{N}, 35.4^{\circ} \mathrm{E}\right)$ on August 1,2009 as indicated with crosses in Figure $1 \mathrm{~b}$.

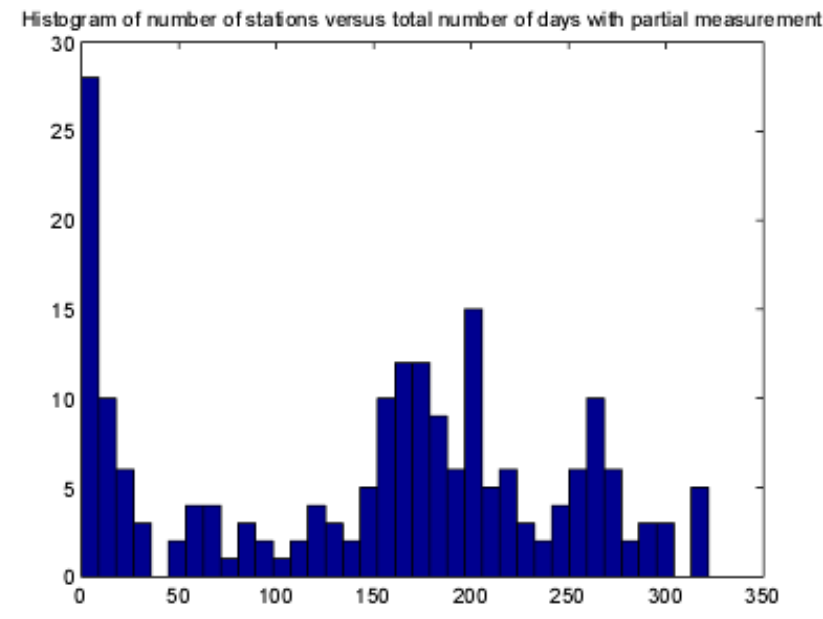

a)

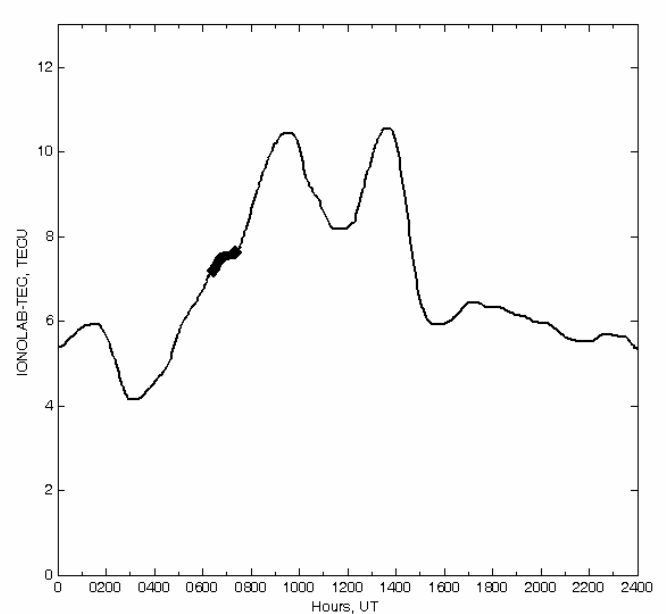

b)

Figure 1: a) Histogram of number of stations versus total number of days with partial measurement in the 2009 in TNPGN b) IONOLAB-TEC for Adana station on August 1, 2009; crosses indicate the region chosen for interpolation

In Figure 2a, the original IONOLAB-TEC estimates are indicated with temporal interpolation only; the spatial interpolation only and the proposed space-time interpolation method. The chosen neighborhood stations are Kilis, Mersin, Gaziantep that are $180 \mathrm{~km}, 80 \mathrm{~km}$ and $200 \mathrm{~km}$ to Adana station, respectively. The interpolation method is tried for 10 different days for the same period of time and the root mean square (RMS) error is indicated in Figure $2 b$. As it can be observed, the proposed method with space-time interpolation has the smallest RMS error for all days.

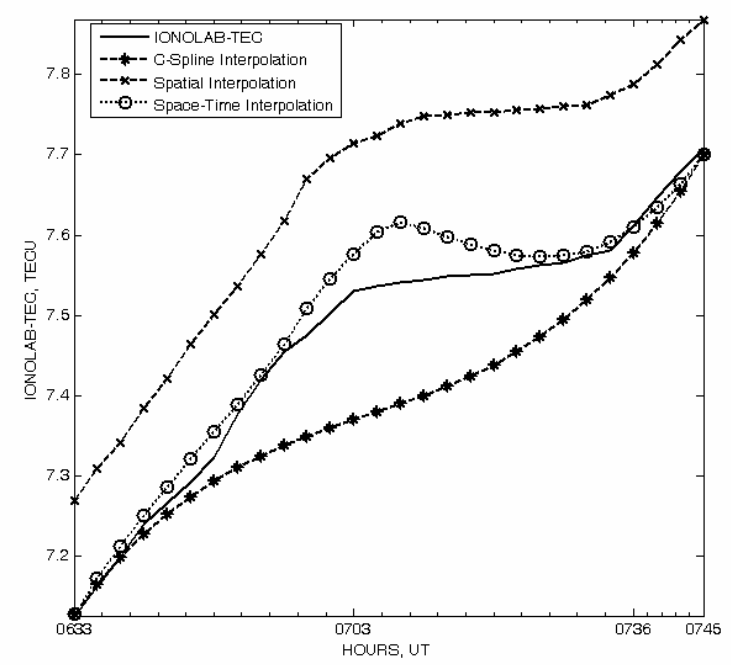

a)

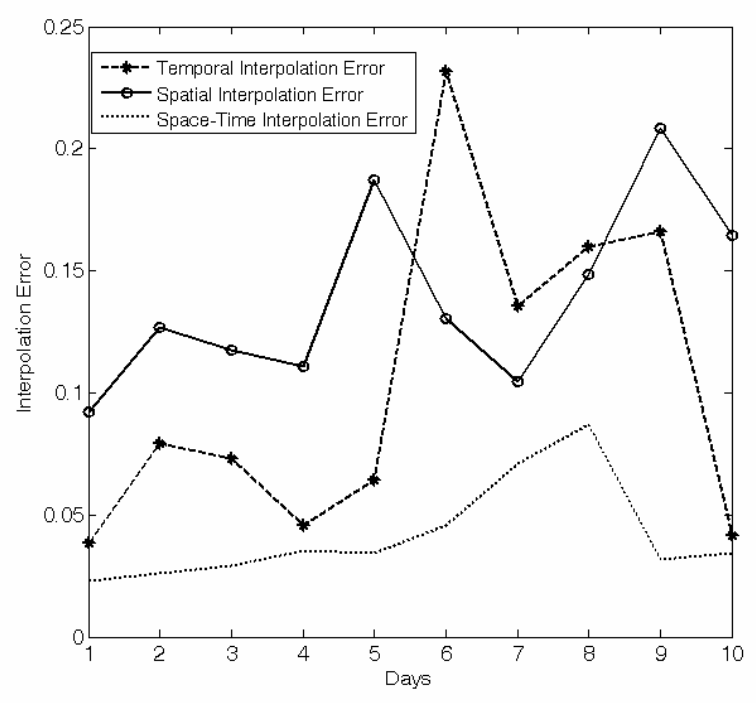

b)

Figure 2: a) IONOLAB-TEC (solid line), and interpolated values with temporal interpolation only (asterisk-dashed line), spatial interpolation only (cross-dashed line) and space-time interpolation (circles-dotted line) b) RMS error for 
10 different days for adan for the same time period; temporal interpolation only (asterisk-dashed line), spatial interpolation only (circles-solid line) and space-time interpolation (dotted line)

\section{Automatic TEC Mapping}

Automatic TEC Maps are generated at optimum Temporal Update Periods (TUP) [13] form an important data base for derivation of space-time random model TEC, monitoring space weather and update of IRI-Plas model [14]. Turkey is located in a mid-latitude region with a predominant east-west extension. IONOLAB group investigates the automatic TEC mapping problem through the automatic generation of theoretical semi-variogram function for Ordinary Kriging method [5]. Making use of synthetic TEC surfaces similar to those in [5-6], reveals that the trend in TEC distribution is the most important factor in the semivariogram function. Also, the semivariogram function for TEC such as surfaces deviates from those functions that are widely used in geophysics and mining. In Figures $3 \mathrm{a}$ and $3 \mathrm{~b}$, TEC maps of Turkey are provided at 1200 UT for July 4, 2009 (quiet day) and July 22, 2009 (positively disturbed day), respectively. The black dots represent the CORS-TR stations. These maps are generated by Ordinary Isotropic Kriging. An east-west linear trend in Figure $3 \mathrm{a}$ is replaced with a north-south more complex trend in Figure 3b. Local anisotropicities can also be observed in both cases and optimum description for anisotropic Ordinary Kriging is currently investigated in TUBITAK project through synthetic TEC surfaces that have various trends and anisotropicities. These studies indicate that local small scale variabilities may be recovered by anisotropic Kriging. In standard Ordinary or Universal Kriging methods, the local variations are overlooked due to the predomination of the trend.

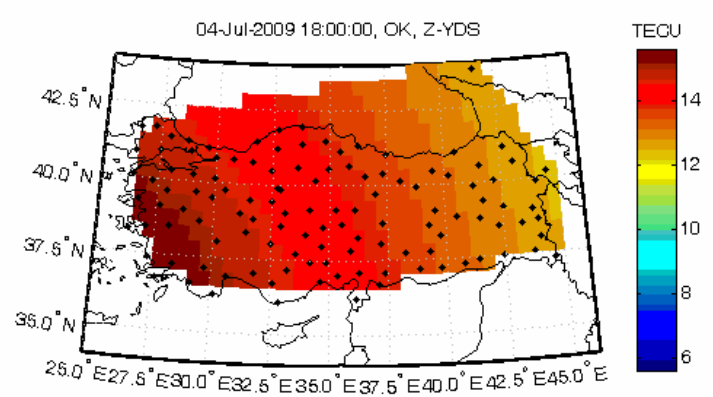

a)

Figure 3: TEC map of Turkey with Isotropic Ordinary Kriging at 1200 UT on a) July 4, 2009 b) July 22, 2009. The black dots indicate CORS-TR stations.

\section{Conclusion and Future Work}

IONOLAB group is currently investigating new methods in space-time interpolation of TEC and automatic mapping of TEC with anisotropic Ordinary Kriging. The developed techniques will be implemented for all available data in TNPGN. The novel space-time interpolation algorithm will be tried for not only quiet, and disturbed days but also for stations that lack data before an earthquake in North Anatolian Fault. These results will form a basis for investigations on Lithosphere-Atmosphere-Ionosphere Coupling models. Theoretical semivariogram functions will be based on particle swarm optimization of the experimental data and a look-up library will be formed. Optimum anisotropic semivariogram functions will also be investigated and appropriate Kriging algorithms will be developed.

\section{Acknowledgments}

This study is sported by TUBITAK EEEAG Grant No: 109E055 


\section{References}

1. F. Arikan, C.B. Erol and O. Arikan,"Regularized estimation of vertical total electron content from Global Positioning System data," Journal of Geophysical Research-Space Physics, 108(A12), 2003, ), pp. 1469 -1480.

2. F. Arikan, C.B. Erol, and O. Arikan,"Regularized estimation of VTEC from GPS data for a desired time period," Radio Science, 39(6), RS6012, 2004.

3. H. Nayir, F. Arikan, O. Arikan, and C. B. Erol, "Total Electron Content Estimation with Reg-Est”, J. Geophys. Res Space Physics, 112(A11313), 2007, pp. 1-11.

4. F. Arikan, H. Nayir, U. Sezen and O. Arikan, "Estimation of single station interfrequency receiver bias using GPSTEC," Radio Science, 43, RS4004, 2008, pp. 1-13.

5. I. Sayin, F. Arikan, and O. Arikan, "Regional TEC Mapping with Random Field Priors and Kriging," Radio Science , 43, RS5012, 2008, pp. 1-14.

6. Yilmaz, A., K. E. Akdogan, and M. Gurun, "Regional TEC mapping using neural networks," Radio Science, 44, RS3007, 2009.

7. O. Arikan, F. Arikan, C.B.Erol, “3-D Ionospheric Tomography with Random Field Priors,” in K. Tas, J.A. Tenreiro Machado and D. Baleanu (eds), Mathematical Methods in Engineering, Netherlands, Springer, 2007, pp. 335-334.

8. O. Arikan, F. Arikan, and C.B. Erol, "Computerized ionospheric tomography with the IRI model," Advances in Space Research, 39, 2007, pp. 859-866.

9. O. Erturk, O. Arikan, F. Arikan, "Tomographic Reconstruction of the Ionospheric Electron Density as a Function of Space and Time," Advances in Space Research, 43, 2009, pp. 1702-1710.

10. C.B. Erol and F. Arikan, "Statistical analysis of the ionosphere using GPS signals," Journal of Electromagnetic Waves and Applications, 19(3), 2005, pp. 373 - 387.

11. N. Turel and F. Arikan, "Probability density function estimation for characterizing hourly variability of ionospheric total electron content," Radio Science, 45, RS6016, 2010, pp. 1-10.

12. S. Karatay, F. Arikan, O. Arikan, "Investigation of total electron content variability due to seismic and geomagnetic disturbances in the ionosphere”, Radio Science, 45, RS5012, 2010, pp. 1-12.

13. I. Sayın, F. Arikan, K.E. Akdogan, “Optimum temporal update periods for regional ionosphere monitoring," Radio Science, 45, RS6018, 2010, pp. 1-9.

14. T.L. Gulyaeva, "Storm time behavior of topside scale height inferred from the ionosphere-plasmasphere model driven by the F2 layer peak and GPS-TEC observations," Advances in Space Research, doi: 10.1016/j.asr.2010.10.025, 2010. 